NIST Special Publication 1200-26

\title{
Assessing the chemical and colloidal stability of functionalized gold nanoparticles
}

Tae Joon Cho

Vincent A. Hackley

This publication is available free of charge from:

https://doi.org/10.6028/NIST.SP.1200-26

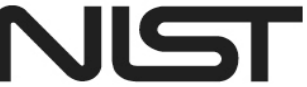

National Institute of Standards and Technology

U.S. Department of Commerce 
NIST Special Publication 1200-26

\section{Assessing the chemical and colloidal stability of functionalized gold nanoparticles}

Tae Joon Cho

Vincent A. Hackley

Materials Measurement Science Division

Material Measurement Laboratory

This publication is available free of charge from:

https://doi.org/10.6028/NIST.SP.1200-26

June 2018

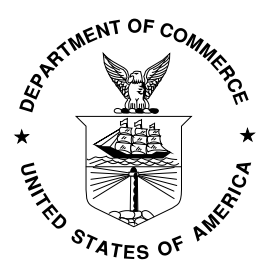

U.S. Department of Commerce Wilbur L. Ross, Jr., Secretary

National Institute of Standards and Technology Walter Copan, NIST Director and Under Secretary of Commerce for Standards and Technology 
Certain commercial entities, equipment or materials may be identified in this document in order to describe an experimental procedure or concept adequately. Such identification is not intended to imply recommendation or endorsement by the National Institute of Standards and Technology, nor is it intended to imply that the entities, materials or equipment are necessarily the best available for the purpose.

Publications in the SP 1200 subseries include written procedural methods in the design and implementation of experiments that ensure successful replication of results by others. Publications may include detailed procedures,

lists of required equipment and instruments, information on safety precautions, the calculation of results and reporting standards.

National Institute of Standards and Technology Special Publication 1200-26

Natl. Inst. Stand. Technol. Spec. Publ. 1200-26, 17 pages (June 2018)

CODEN: NSPUE

This publication is available free of charge from: https://doi.org/10.6028/NIST.SP.1200-26

Please direct all communication regarding this publication to the corresponding author:

Tae Joon Cho (tjcho@nist.gov) 


\section{FOREWORD}

The 1200 series of Special Publications originated in response to the National Nanotechnology Initiative (NNI) Nanotechnology Environmental Health and Safety Research Strategy published in 2011, which identified Nanomaterial Measurement Infrastructure as one of the essential areas of research needed in order to develop an effective risk assessment and management plan regarding various aspects of nanotechnology in consumer products as it pertains to human health, exposure and the environment. The National Institute of Standards and Technology (NIST) was identified as a lead agency in the development of measurement strategies to address this need. The protocols developed by NIST and its partners address a wide range of measurement issues related to the design, production, evaluation, quality control and safety of nanomaterials and products that contain them. These protocols are intended for use by industry, researchers and regulatory agencies to support safe and sustainable development of nanotechnology.

This protocol describes a series of methods to assess the chemical and physical (colloidal) stability of functionalized gold nanoparticles, particularly with respect to their potential use in biomedical applications such as nanomedicine. The methods described herein have been previously validated and published in the peer reviewed scientific literature. Updates to this protocol may be released in the future. Visit https://www.nist.gov/mml/nano-measurementprotocols to check for revisions of this protocol, or new protocols in the 1200 series. We encourage users to report citations to published work in which this protocol has been applied and to provide feedback on its application.

\section{Keywords}

gold; functional coating; nanoparticles; chemical stability; physical stability 


\section{Introduction}

Among the many classes of engineered nanomaterials, gold nanoparticles (GNPs) are one of the most widely studied and utilized nanoscale platforms for biomedical applications including diagnostics, therapeutics, and transfection. ${ }^{1,2}$ This is due in part to their general biocompatibility, facile synthesis and unique optical properties. ${ }^{3}$ To realize these achievements, a systematic evaluation of GNP stability, particularly under physiologically relevant conditions, is critical for the efficacious and safe application of GNPs in any biomedical or technical application. A lack of well documented and reproducible protocols motivated the development of the present collection of methods, which address stability with respect to aging, different media, $\mathrm{pH}$, temperature, chemical resistance, and long-term storage.

\section{Principles and scope}

Size and/or size distribution, ${ }^{4}$ and the surface plasmon resonance (SPR) peak ${ }^{5}$ are fundamental and characteristic features to determine the relationship between the colloidal behavior and stability of GNPs. In this protocol, GNPs are dispersed in appropriate media associated with specific conditions. Two major properties, size/size distribution and SPR absorption of test materials are measured initially and over a specified period of time under each specified set of conditions by dynamic light scattering (DLS) ${ }^{6}$ and UV-Vis spectrophotometry, respectively. With respect to size distribution, increased size or a broadened distribution indicates the presence of agglomeration/aggregation due to a decrease in colloidal stability. ${ }^{7-9}$ Similarly, a red-shift in the SPR peak can be further evidence for morphological transformation of GNPs due to inter-particle contact (agglomeration) and/or core-to-core fusion (aggregation). ${ }^{7-9}$ Moreover, reduction in the magnitude of the SPR peak is evidence for either direct and rapid precipitation leading to settling of GNPs or dissolution of GNPs resulting from a lack of chemical resistance. ${ }^{7,8}$ Based on the results of a systematic application of this protocol, the quality and appropriateness for use of the test material can be evaluated.

\section{Key parameters for physiologically relevant stability tests}

The key parameters for test conditions are as follows.

\subsection{Shelf-life (aging)}

Shelf-life or aging is an important factor for utilization of GNPs as reference or research materials. Typically, a stability of 6 months to 1 year under ambient laboratory conditions is considered sufficient for most applications.

\subsection{Stability in relevant media}

Evaluation of GNP stability in different media, including physiologically relevant media, is critical for an accurate assessment of their appropriateness for many biomedical applications. Media include deionized water, salt solutions, buffers, and cell culture media with and without serum proteins, since serum contains thiolated proteins that can potentially replace the native surfacebound ligands, proteins or nucleic acid segments/DNA.

\section{3. $\mathrm{pH}$}


The $\mathrm{pH}$ dependent stability is an important consideration for therapeutic applications of administered GNPs, as well as other potential biomedical uses. For instance, highly acidic conditions exist in the stomach (approaching $\mathrm{pH} 2$ ), while proceeding into and through the intestines, the $\mathrm{pH}$ gradually reaches mildly basic conditions $(\approx \mathrm{pH} 8)$. Lysosomes within mammalian cells are characterized by moderately acidic $\mathrm{pH}$ levels (between 4 and 5), and the $\mathrm{pH}$ of the blood stream is generally neutral (7.4). Hence, particles can experience a broad range of conditions within a biological system, ranging from very acidic to mildly alkaline. ${ }^{10}$

\subsection{Temperature}

Thermal stability of GNPs should be evaluated over a range that includes ambient, physiological and higher temperatures (typically (20 to 60) ${ }^{\circ} \mathrm{C}$ ) that cover the relevant range for storage, transport, biological assays and most biomedical applications.

\subsection{Chemical resistance}

Nanomaterial formulations intended for therapeutic applications are most often administered intravenously. An appropriate hydrophilic and stable functionalized GNP will minimize nonspecific protein binding, reduce immune system recognition and removal, and prolong systemic circulation to facilitate localization in targeted tissue through enhanced permeability and retention. An ideal GNP platform should have a stable coating resistant to chemical attack and ligand displacement (disruption of surface functionalization).

The cyanide ion $\left(\mathrm{CN}^{-}\right)$acts as a strong complexing ligand for $\mathrm{Au}(\mathrm{I})$ ions, to the extent that addition of KCN rapidly etches Au surfaces on the basis of Elsner's reaction: ${ }^{11}$

$$
4 \mathrm{Au}+8 \mathrm{CN}^{-}+\mathrm{O}_{2}+2 \mathrm{H}_{2} \mathrm{O} \rightarrow 4 \mathrm{Au}(\mathrm{CN})_{2}{ }^{-}+4 \mathrm{OH}^{-}
$$

Digestion of $\mathrm{Au}$ colloids by cyanide ions is therefore a useful means to evaluate the relative chemical resistance of GNP conjugates. When $\mathrm{CN}^{-}$can access the GNP surface, the characteristic ruby-red color of stable GNP suspensions gradually changes to a colorless solution by formation of the soluble $\mathrm{Au}(\mathrm{CN}) 2^{-}$complex.

\subsection{Long-term storage (lyophilization)}

Dry storage (generally obtained by freeze-drying or lyophilization) is often more suitable for longterm storage of sensitive nanomaterials. Subjecting GNPs to a lyophilization-reconstitution process is a useful way to evaluate the potential of this approach for the native material. In a pharmaceutical formulation, excipients (i.e., cryoprotectants and lyoprotectants) would most likely be included, and the method described here can be applied to such formulations. Common excipients include sugars or polyols, polymers, and surfactants. ${ }^{12}$

\section{Reagents and equipment}

\subsection{Reagents}

4.1.1. About (5 to 50) $\mathrm{mg} / \mathrm{kg}$ of aqueous (aq) GNP dispersions as test materials

4.1.2. Deionized (DI) water; at least $18 \mathrm{M} \Omega \bullet \mathrm{cm}$ resistivity, passed through a $0.2 \mu \mathrm{m}$ sterile filter

4.1.3. $0.05 \mathrm{~mol} / \mathrm{L}$ ef $\mathrm{HCl}(\mathrm{aq})$ and $0.05 \mathrm{~mol} / \mathrm{L} \mathrm{NaOH}(\mathrm{aq})$ reagent grade solutions for $\mathrm{pH}$ adjustment 
4.1.4. Phosphate buffered saline (PBS) 10x solution (e.g., HyClone Laboratories, Cat. No. SH30258.02) with $0.067 \mathrm{~mol} / \mathrm{L}\left(\mathrm{PO}_{4}{ }^{3-}\right)$, calcium and magnesium free, passed through a $0.2 \mu \mathrm{m}$ sterile filter following dilution

4.1.5. Dulbecco’s Modified Eagle’s Medium (DMEM); 4.5 g/L glucose and sodium pyruvate without L-glutamine and phenol red, sterile (e.g., Mediatech, Inc., Cat. No. 17-205-CV)

4.1.6. Bovine serum albumin (BSA); 99+ \% high purity reagent grade (lipid and IgG free) powder (e.g., SeraCare Life Science, Product Code AP-4510-80)

4.1.7. $2 \mathrm{mmol} / \mathrm{L}(\mathrm{aq})$ potassium cyanide (KCN) solution; Prepared in the laboratory on demand using reagent grade KCN salt (e.g., Sigma-Aldrich, Cat. No. 207810) and DI water at room temperature to minimize formation of volatile HCN.

Note: Safety guidance is beyond the scope of this protocol, but it should be emphasized that KCN is a highly toxic substance. KCN in aqueous solution may produce volatile, highly toxic HCN, particularly when KCN is combined with acidic solutions. Users are cautioned to protect against all hazards by implementing applicable safety precautions prescribed by local chemical hygiene programs and regulatory requirements. Such precautions should include and may not be limited to: review of associated safety data sheets; use of a properly functioning chemical fume hood; use of appropriate personal protective equipment; use of safe handling practices or specified procedures; completion of any required training; and following local requirements for safe storage and disposal of waste. For further information on hazards and safe handling procedures for KCN, refer to the NIOSH Pocket Guide to Chemical Hazards (https://www.cdc.gov/niosh/npg/npgd0522.html) and the American Chemical Society's Identifying and Evaluating Hazards in Research Laboratories Table F6

(https://www.acs.org/content/dam/acsorg/about/governance/committees/chemicalsafety/publicati ons/identifying-and-evaluating-hazards-in-research-laboratories.pdf).

\subsection{Equipment}

4.2.1. Ultraviolet-visible spectrophotometer (UV-Vis) (e.g., Perkin-Elmer, Lambda 750 with 8+8 cell holder/recirculated water jacket)

4.2.2. Dynamic light scattering (DLS) instrument (e.g., Malvern Panalytical Zetasizer Nano ZS)

4.2.3. Water purification system for DI water (e.g., Aqua Solutions, Type I)

4.2.4. $\mathrm{pH}$ meter and appropriate $\mathrm{pH}$ electrode (e.g., Mettler Toledo, InLab Semi-Micro Combination $\mathrm{pH}$ electrode)

4.2.5. Lyophilizer (Freeze-dryer; e.g., LabConco, FreeZone 6) with temperature programmable stoppering tray dryer (e.g., LabConco, Model 7948020)

4.2.6. Temperature controllable recirculating bath (e.g., VWR, Model 1160-S)

4.2.7. Analytical balance with readability to $0.1 \mathrm{mg}$ (e.g., Mettler Toledo, MS 104S)

4.2.8. Optical quality cuvettes (quartz, glass, or disposable (polymethylmethacrylate; PMMA)) for UV-Vis and (quartz, glass or disposable PMMA or polystyrene) for DLS measurements; same cuvette can be used for both measurements if compatible 
4.2.9. Calibrated pipettes and sterile disposable tips covering a (0.020 to 5.000) $\mathrm{mL}$ range; adjustable volume pipettes are recommended

4.2.10. 5-mL and 10-mL transport tubes (e.g., Axygen Scientific sterile polypropylene tubes with screw caps)

4.2.11. 6-mL glass vials (e.g., Wheaton Shorty Vials) and 10-mL serum bottles (e.g., LabConco, Part \#7573210) with two-leg rubber septums (e.g., LabConco, Part \#7576210)

\section{General aspects of protocol}

\subsection{Sample preparation}

Samples and test media were prepared, diluted and combined in a Class II, Type A2 Biosafety Cabinet (e.g., Labconco, Purifier Logic series) to protect both worker and sample from contamination and to maintain sterility. While not required for this protocol, the use of such a device is highly recommended. The final concentration of GNPs in test suspensions, referred 'test concentration', should be in the range from about (5 to 50$) \mathrm{mg} / \mathrm{kg}$, for DLS and UV-Vis measurements. Concentrations of test materials are obtained from vendor's information for commercially available GNPs and by ICP-MS for in-house synthesized GNPs. For a stability comparison test of different types of GNPs, the concentration of each GNP sample in a medium should be identical within a range of recommended test concentration.

\subsection{Measurements}

DLS procedures used in this protocol and for validation and demonstration follow the National Institute of Standards and Technology (NIST) - Nanotechnology Characterization Laboratory (NCL) protocol ${ }^{6}$ (freely available online) with z-average values reported as the mean of no less than five measurements plus or minus one standard deviation. Disposable optical quality microcuvettes were used for DLS measurements. UV-Vis spectra were collected using UV-transparent optical quality cuvettes with a $1 \mathrm{~cm}$ path-length, and requiring $1 \mathrm{~mL}$ to fill the light path of the UV-Vis spectrometer. All DLS and UV-Vis measurements were conducted at $20 \pm 0.1^{\circ} \mathrm{C}$ except thermal stability tests. Sample temperature for UV-Vis measurements was controlled using a water circulator combined with an appropriate cell-holder jacket. DLS temperature was controlled by an on-board Peltier device.

\subsection{Safety}

This document does not purport to address all safety concerns associated with this protocol. It is the responsibility of the user to establish appropriate safety practices according to local requirements or regulatory limitations prior to its use.

\section{Stability tests}

\subsection{Shelf-life (long term aging) test}

Store the test sample under ambient conditions (typically from $20^{\circ} \mathrm{C}$ to $25^{\circ} \mathrm{C}$ away from direct light) for at least 6 months, and periodically monitor size by DLS and absorption by UV-Vis. 
Compare results to those obtained initially from freshly prepared sample (initial measurement should be conducted within $24 \mathrm{~h}$ after preparation/purification if possible or a maximum of 1 week if additional purification steps are required). Use identical conditions for all measurements with respect to dilution factor (concentration) and instrumental parameters (i.e., standard operating procedure - SOP); e.g., temperature, number of runs, measurement duration, or wavelength range). Dilute sample only if necessary for analysis.

\subsection{8 h media stability test}

6.2.1. Preparation of PBS: Prepare $100 \mathrm{~mL}$ of PBS by performing a 1:10 dilution of the stock PBS10x with DI water, i.e., add $10 \mathrm{~mL}$ of PBS10x to $90 \mathrm{~mL}$ of DI water, mix thoroughly and pass through a $0.2 \mu \mathrm{m}$ sterile filter.

6.2.2. Preparation of $10 \%$ mass fraction BSA in DMEM: Place $1 \mathrm{~g}$ of BSA powder into 10-mL transport tube and add $9 \mathrm{~g}$ of DMEM solution. Shake mixture to allow complete dissolution and store for about (1 to 2 ) h to minimize foam formation, then pass through a $0.2 \mu \mathrm{m}$ sterile filter before use.

\subsubsection{Stability test}

Stability in different aqueous media, including physiologically relevant media, is tested over a 48h period (relevant to many cell-based in vitro assays). Turn on and set up the DLS and UV-Vis instruments with appropriate SOPs for measurements prior to sample preparation. Once instruments are set up, disperse (dose) test materials into the selected medium within the recommended test concentration range (5.1.) and measure immediately to evaluate initial behavior of test materials in a specific medium. After the first set of measurements, measure every ' $n$ ' hour (interval depends on requirements) over $48 \mathrm{~h}$ with identical SOP. For GNP $\geq 60 \mathrm{~nm}$ diameter, invert cuvette several times or mildly agitate before each measurement to avoid settling.

\subsection{2 h pH stability test}

For purposes of testing the relative chemical/physical stability of GNPs over a desired pH range, $12 \mathrm{~h}$ is suitable for most applications. Each $\mathrm{pH}$ solution is prepared by mixing $50 \mathrm{mmol} / \mathrm{L} \mathrm{HCl}$ and $50 \mathrm{mmol} / \mathrm{L} \mathrm{NaOH}$ to adjust test solution to the required $\mathrm{pH}$. For example, to $10 \mathrm{~mL}$ of $0.05 \mathrm{~mol} / \mathrm{L}$ $\mathrm{HCl}(\mathrm{NaOH})$ equipped with stir bar and magnetic stirrer, add dropwise $0.05 \mathrm{~mol} / \mathrm{L} \mathrm{NaOH}(\mathrm{HCl})$ step by step until desired $\mathrm{pH}$ is obtained as measured using a $\mathrm{pH}$ electrode. Use a combination $\mathrm{pH}$ electrode and meter that have been calibrated according to vendor instructions. Prepare and analyze samples in a manner similar to section 6.2.3.; i.e., GNP is dispersed into $\mathrm{pH}$ solution and measured by DLS and UV-Vis immediately for evaluation of initial behavior, then measured or recorded every ' $n$ ' minutes (interval depends on requirements) over $12 \mathrm{~h}$ period. Initial and final $\mathrm{pH}$ values should be reported.

\subsection{Thermal stability test}

Temperature dependent stability of GNPs should be evaluated by DLS and UV-Vis absorbance measurements over the range from (20 to 60$){ }^{\circ} \mathrm{C}$. In general, for the thermal stability test, samples are dispersed in DI water in the recommended test concentration range (5.1) and incubated for 30 min at each temperature before measurements are conducted. Temperature increments can vary, but should be no larger than $5{ }^{\circ} \mathrm{C}$ (e.g., $20^{\circ} \mathrm{C}, 25^{\circ} \mathrm{C}, 30^{\circ} \mathrm{C}, 35^{\circ} \mathrm{C}$, etc.). The inclusion of a data point for $37^{\circ} \mathrm{C}$ is recommended due to its human physiological relevance. 


\subsection{Chemical resistance test}

The chemical stability of GNPs is evaluated by examining the resistance to cyanide etching. ${ }^{11}$ Prior to sample preparation, set up the UV-Vis instrument for kinetic operation (i.e., measure at ' $n$ ' time intervals at a fixed wavelength). The test GNPs are then rapidly diluted (within recommended test concentration range) into (aq) KCN (0.002 $\mathrm{mol} / \mathrm{L})$, and measurement of the dissolution rate is begun immediately by monitoring the optical absorbance (optical density). The typical volume for this test is $1 \mathrm{~mL}$. The measurement interval can vary from seconds to hours depending on the test materials and desired time frame of the experiment. For GNPs, a wavelength of $520 \mathrm{~nm}$ is generally recommended since it is close to the SPR peak for spherical GNPs from $10 \mathrm{~nm}$ to $60 \mathrm{~nm}$ in diameter. However, the wavelength can also be set to match the initial SPR peak (determined from a wavelength scan) for that particular GNP sample; the SPR maximum value will depend on size and its use may improve sensitivity to change. Regardless, for a given GNP core size, the same wavelength should be used for comparison.

\subsection{Lyophilization-reconstitution test}

Place $2 \mathrm{~mL}$ of original suspension (4.1.1., e.g., in DI water) of the test material into a 6-mL vial (e.g., Wheaton borosilicate vials) or a 10 -mL serum bottle, and freeze rapidly by immersing the vial/bottle into liquid nitrogen in a Dewar flask. Attach vial/bottle to the freeze drier and operate the freeze drier for about $36 \mathrm{~h}$ following the vendor's instructions. Seal sample until needed. After lyophilization, reconstitute the dried sample in a volume of DI water identical to the original suspension, store overnight at ambient temperature and measure the following day with DLS and $\mathrm{UV}$-Vis to compare before and after the lyophilization cycle. The cycle as described here is processed without the aid of excipients; however, this test can be repeated in the presence of excipients if desired.

\section{Analysis}

\subsection{DLS analysis}

From DLS one obtains the intensity-weighted equivalent hydrodynamic diameter $\left(d_{\mathrm{h}}\right)$ associated with the test materials. Briefly, DLS measures the diffusion coefficient $\left(D_{z}\right)$ of particles undergoing random thermal motion, and by application of the Stokes-Einstein relationship (equation 1) $d_{\mathrm{h}}$ is obtained. ${ }^{4}$

$$
d_{h}=\frac{k T}{3 \pi \mu D_{z}}
$$

Here, $k$ is Boltzmann's constant, $\mu$ is viscosity of the medium, and $T$ is the absolute temperature of measurements.

\subsubsection{Size}

As mentioned above, the NIST-NCL protocol $^{6}$ recommends reporting z-average diameter (or radius) along with one standard deviation for the size of the test materials. The z-average size is obtained by application of the cumulants method, ${ }^{14}$ which assumes a single size Gaussian mode. This approach works best for monomodal populations that are not highly polydisperse. 


\subsubsection{Size distribution}

Derive a size distribution by application of a suitable inversion algorithm with appropriate smoothing in combination with non-negative least squares (NNLS) fitting to the measured correlation function. The intensity-weighted size distribution should be used for comparative purposes only. In this case, the objective is to detect changes in the apparent size distribution and not necessarily to obtain absolute accuracy.

\subsubsection{Polydispersity index (PdI)}

The PdI value is an effective indicator of how narrow or broad the size distribution of a test material is, but it is at best a rough estimate and, like the z-average size, is derived from the cumulants analysis. In general, high quality commercially available nanomaterials will exhibit a PdI value $\approx$ 0.1 to 0.2 . Values below 0.1 are considered monodisperse, and are typically obtained only with monodisperse polystyrene beads or similar standards of extremely high quality.

\subsection{UV-Vis analysis}

The UV-Vis absorbance spectrum for GNPs typically shows an SPR peak near $\lambda_{\max } \approx 520 \mathrm{~nm}$ (this value can vary from slightly below $520 \mathrm{~nm}$ to more than $535 \mathrm{~nm}$, depending on the GNP diameter). ${ }^{5}$ The observance of a red shift in the SPR peak suggests formation of particle agglomerates or aggregates and its absence suggests resistance to agglomeration during a GNPconjugate formation process ${ }^{15}$ or stability test. ${ }^{7-9}$

\section{Interpretation of stability test results}

Interpretation of stability test results is a discussion of the behavioral changes of the test materials before and after being subjected to potentially perturbating conditions based on measurement results and observations. DLS measurements provide information on the change or resistance to change of the hydrodynamic size $\left(\Delta d_{\mathrm{h}}\right)$ and/or size distribution (modality) of the test materials. Positive $\Delta d_{\mathrm{h}}$ values after a test indicates that the test materials have agglomerated or aggregated. Not only mean size change, but also a broadened size distribution (occasionally with multimodality) indicates the perturbating factor (time, temperature, $\mathrm{pH}$, matrix, etc.) has resulted in morphological transformation. This transformation suggests loss of, or change in, the chemical and/or physical stability of the GNPs.

Moreover, a change in the SPR peak position or intensity measured by UV-Vis absorbance is another indicator of the stability or lack thereof for the test material. The appearance of new absorbance peak(s) at higher wavelengths or the red-shifted SPR band $\left(+\Delta \lambda_{\max }\right)$ for the test material after exposure to a certain test condition or perturbation indicates the occurrence of a size increasing event. Broadening of the SPR band relative to the initial spectrum is also evidence of a broadening size distribution. Additionally, if the optical density of the SPR peak is reduced over time without an associated red-shift, this observation suggests removal of the test material from the detection zone, probably as a result of agglomeration/aggregation followed by rapid sedimentation or coating on the side walls of the cuvette (i.e., loss of material to the cell) during measurements. This observation should be validated by comparison with DLS results on the same material. On the other hand, the reduction of the SPR peak intensity without the occurrence of sedimentation/coating/size increase, but associated with the overall disappearance of color, would suggest the core GNP is undergoing dissolution, for instance due to $\mathrm{CN}^{-}$etching as described previously. 


\section{Practical examples and validation}

\section{Example 1. Shelf-life (long term aging) test}

Figure 1 shows the long term (2 to 6 months) stability for three different GNP-conjugates. Each GNP sample exhibits distinct long-term stability as a result of the type of surface conjugation. The aged samples in (b) and (c) yield nearly identical mean size and size distributions compared with the initial, freshly prepared and purified products (see top row of Figure 1, DLS results). In contrast, the size distribution for sample (a) significantly shifts toward larger sizes over 2 months, indicating this sample is less stable compared with the other GNP samples. Note that the size distributions are shown on a log scale.

The associated SPR peak intensities and positions (UV-Vis, bottom row of Figure 1) provide a more sensitive probe to changes in the near-field environment of the GNPs. The substantially redshifted ( $\Delta \lambda_{\max } \approx 10 \mathrm{~nm}$ ) absorbance maximum for sample (a) suggests a large increase in size via agglomeration followed by sedimentation. GNPs (b) and (c) show minor red shifts with $\Delta \lambda_{\max } \approx 2$ $\mathrm{nm}$, in both cases. Based on these test results, the long-term stability of these GNP formulations can be differentiated, and the optimum material selected for further tests.
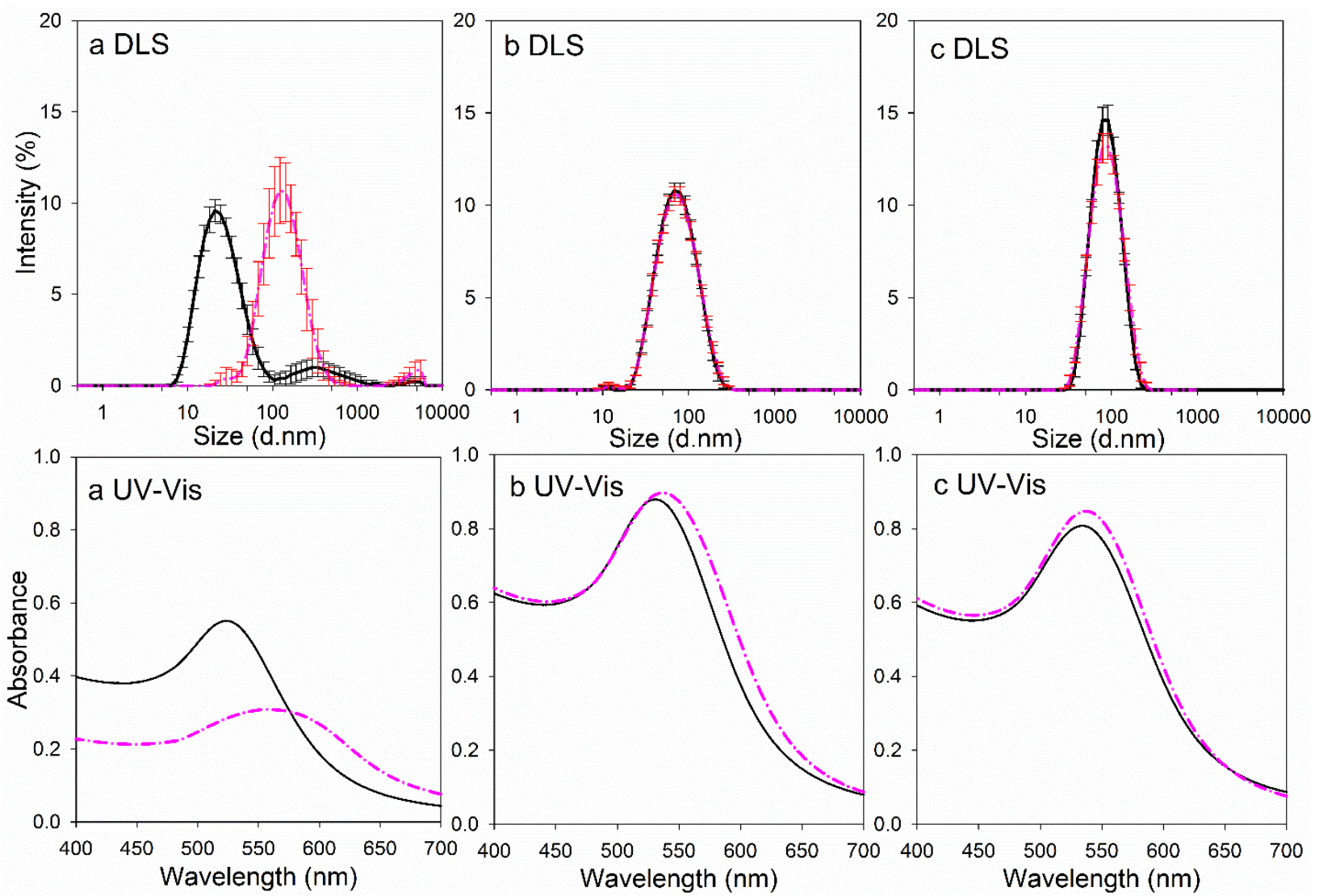

Figure 1. Shelf-life study of polymer stabilized GNPs (molar mass of ligand (polymer); $\mathrm{a}<\mathrm{b}<\mathrm{c}$ ) examined by DLS (top row) and UV-Vis (bottom row) in DI water. Black solid line: initially purified samples; pink dotted line: after 2 months for GNPs (a), and 6 months for GNPs (b) and (c). Error bars represent the standard deviation of 5 replicate measurements. ${ }^{9}$ 


\section{Example 2. $48 \mathrm{~h}$ media stability test}

Thiolated dendron stabilized GNPs diluted into PBS exhibit excellent stability over $48 \mathrm{~h}$ based on hydrodynamic size and absorbance spectra (see Figure 2a). This behavior is attributed to (1) the hydrophilicity of the PEG segment inserted into the dendron structure and (2) dendritic steric repulsive interactions that substantially reduce salt sensitivity (from screening of electrostatic charge). ${ }^{7}$ The results for stability in the more complex DMEM matrix over $48 \mathrm{~h}$ (see Figure 2b) are less obvious. The mean size as determined by DLS is largely constant over the time period of the test, but the SPR band intensity decreases (without red-shifting) by about $20 \%$ over the same period. Normally, the reduction in the SPR absorbance would be attributed to removal of material, perhaps by agglomeration followed by rapid sedimentation. This cannot be ruled out here, but the constant DLS size and absence of red-shift in the SPR peak suggests an incipient reaction between the test material and the DMEM components that leads to the loss of SPR enhanced absorbance without impacting stability. ${ }^{8}$ Notably, $10 \mathrm{~nm}$ nominal citrate stabilized GNPs (cit-GNPs) show extremely unstable behaviors and are precipitated (removed) almost immediately in PBS buffer (Figure 2c).

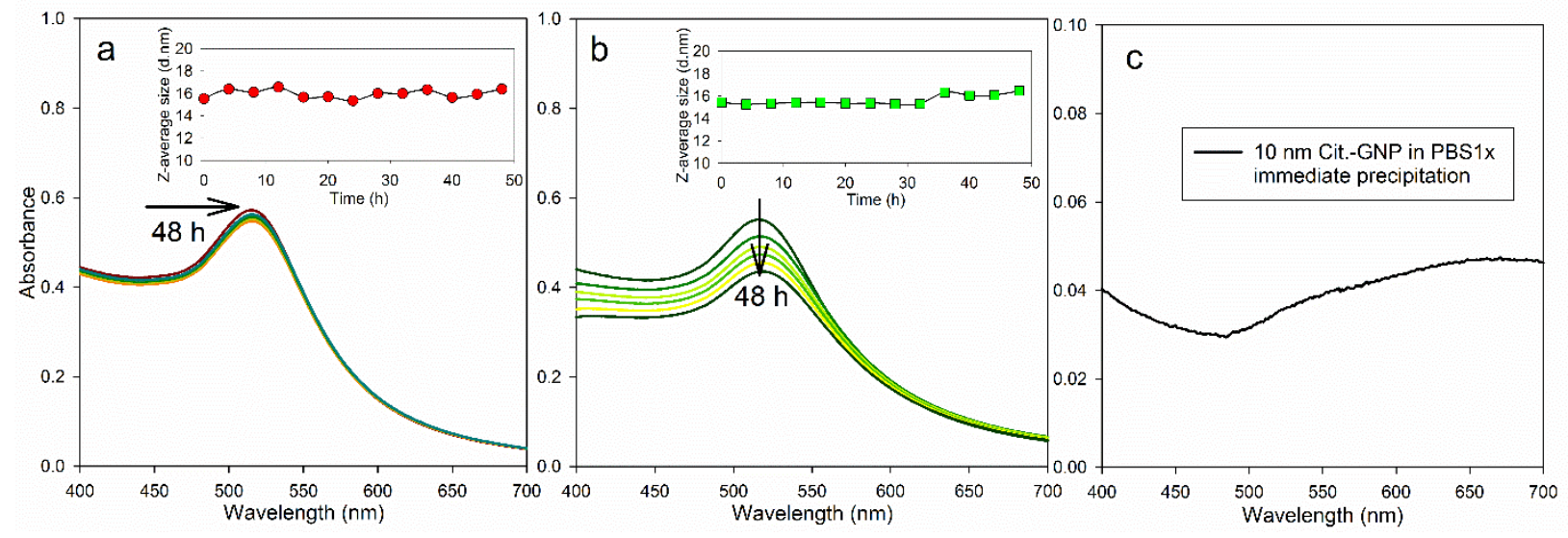

Figure 2. Stability of dendron conjugated GNPs in biological test media over $48 \mathrm{~h}$, as monitored by UV-Vis absorbance and z-average diameter from DLS (inset): (a) PBS, (b) DMEM, ${ }^{8}$ and (c) cit-GNPs in PBS showing rapid precipitation. ${ }^{7}$

\section{Example 3. $12 \mathrm{~h} \mathrm{pH}$ stability test}

At pH 3, cit-GNPs show a continuous increase in mean size with time over $12 \mathrm{~h}$, however, thioldendron encapsulated GNPs show a noticeable size increase initially (within $1 \mathrm{~h}$ of contact with acidic medium) and then exhibit relative stability up to $12 \mathrm{~h}$ (see Figure 3a; DLS). By comparison, UV-Vis spectra for cit-GNPs (see Figure $3 b$ ) exhibit a gradual reduction in the initial SPR peak intensity (at $\approx 520 \mathrm{~nm}$ ), while developing broader absorbance over time at longer wavelengths $(\approx$ 620 to 720$) \mathrm{nm}$. For the surface modified GNPs (see Figure 3c), the SPR peak is red shifted ( $\Delta \lambda_{\max }$ $=25 \mathrm{~nm}$ ) during the initial $1 \mathrm{~h}$ and stabilizes near $560 \mathrm{~nm}$ for up to $12 \mathrm{~h}$. The UV-Vis results generally confirm the DLS results in this case. However, the absorbance in Figure 3c gradually 
decreases in intensity over $12 \mathrm{~h}$, which suggests loss of material, even though the apparent size is relatively stable. Overall, with these results, it can be concluded that thiol-dendron modified GNPs show substantially improved stability in acidic solution compared with cit-GNPs.
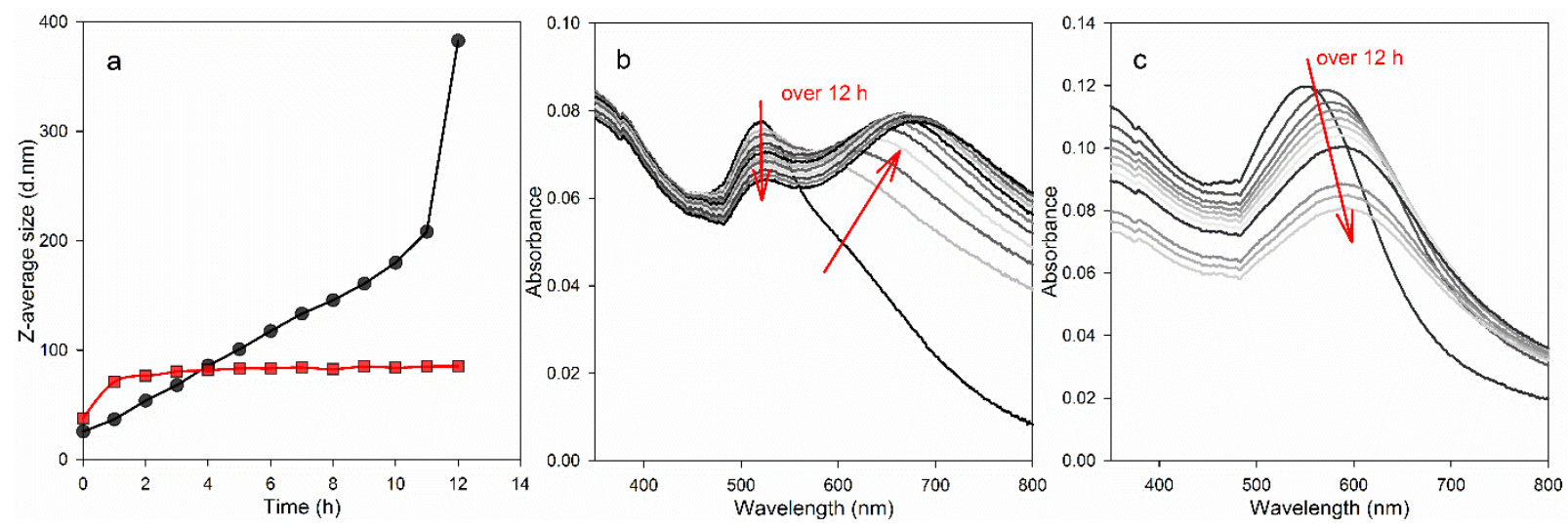

Figure 3. Time dependent stability studies of GNPs in pH 3. By DLS measurements, (a) z-average size versus time for cit-GNPs (nominal $10 \mathrm{~nm}$, black circle) and surface modified (red square) dendron-GNPs. UV-Vis spectra of (b) cit-GNPs and (c) surface modified GNPs over $12 \mathrm{~h}$ at $\mathrm{pH}$ $3 .^{7}$

\section{Example 4. Thermal stability test}

Temperature dependent hydrodynamic size (inset) and SPR absorbance for dendron-GNPs measured by DLS and UV-Vis, respectively, are shown in Figure 4. The constancy of the z-average size (from DLS) and the SPR peak (from UV-Vis spectra) confirm that the GNPs are stable with respect to temperature over the tested range. The slight increase in size is not statistically meaningful.

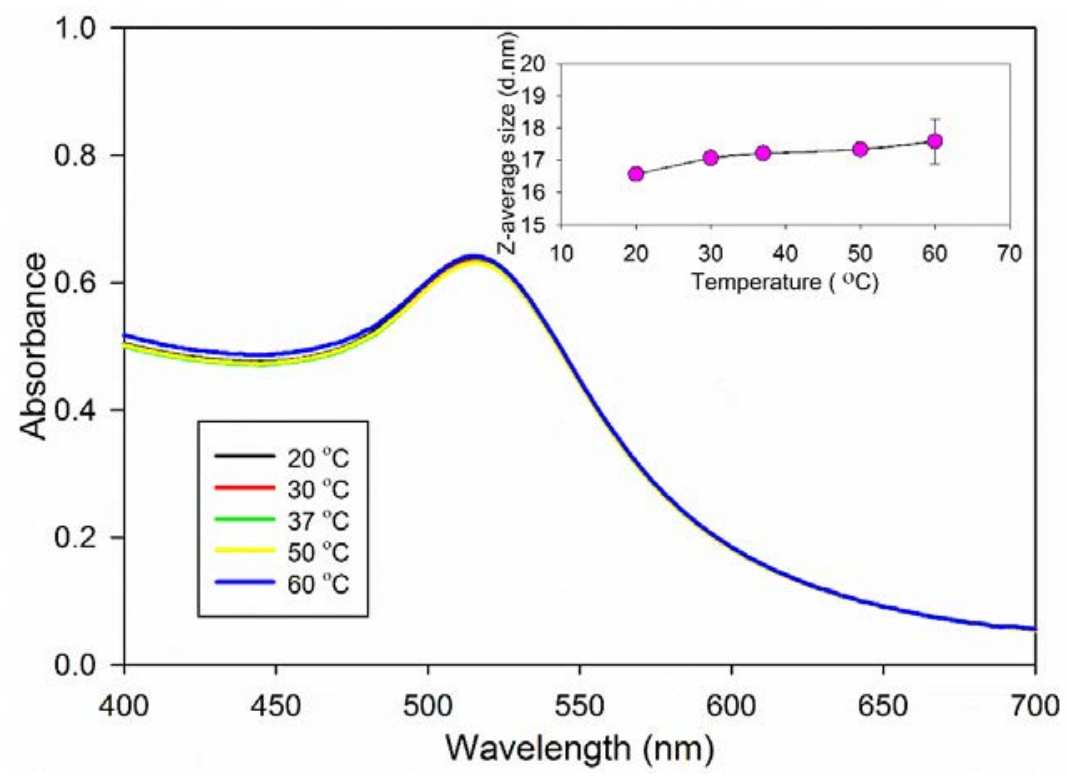

Figure 4. Temperature dependent stability of GNPs by DLS and UV-Vis. ${ }^{8}$ 


\section{Example 5. Chemical resistance test}

The dissolution rate of cit-GNPs in KCN solution (see Figure 5a) exhibits significant dependence on the initial core size. Nominal $5 \mathrm{~nm}$ diameter GNPs show the fastest decay rate (black circle) while $60 \mathrm{~nm}$, the largest GNPs, exhibit the slowest dissolution (blue square). This indicates obviously that larger GNPs need more time (or more $\mathrm{CN}^{-}$ions) for complete oxidation from $\mathrm{Au}^{0}$ to $\mathrm{Au}^{\mathrm{I}}$. Figure $5 \mathrm{~b}$ shows the substantial effect of dendron encapsulation on the dissolution rates of the same size GNPs. The presence of the dendron ligand provides chemical resistance due to blockage of the GNP surface and/or steric repulsion (branching effect) against the attack of ionic chemical species. The relative rates correlate to chemical resistance, where the cit-GNPs serve as the positive control.
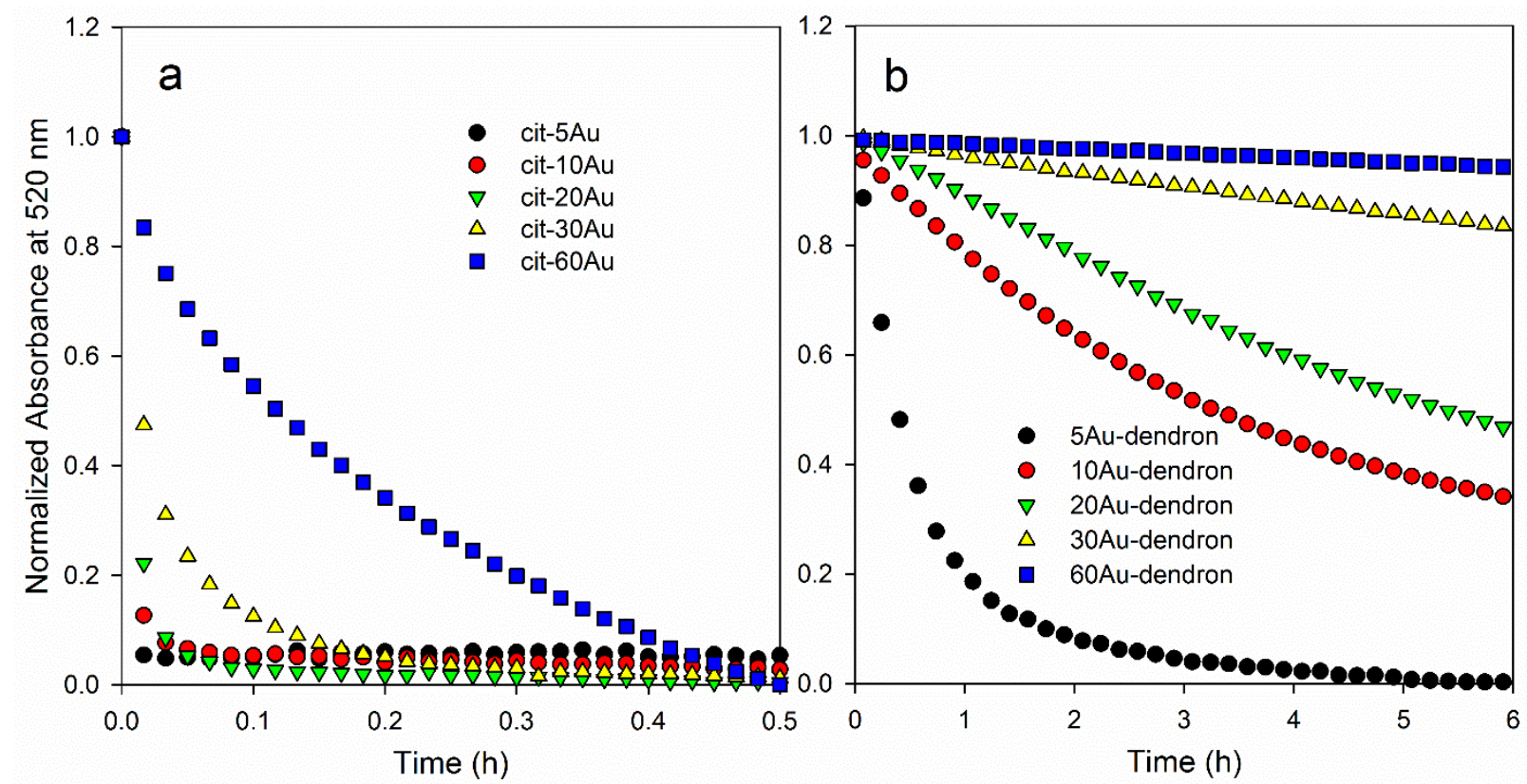

Figure 5. Normalized absorbance decay at $520 \mathrm{~nm}$ for various core size of (a) cit-GNPs and (b) denron-GNPs following treatment with $2.0 \mathrm{mmol} / \mathrm{L} \mathrm{KCN}$ solution.

\section{Example 6. Lyophilization-reconstitution test}

In Figure 6, analysis of reconstituted dendron conjugated GNPs lyophilized (without the aid of excipients) exhibit excellent recovery of the primary NP size, with a 5-nm shift in the z-average value. Evidence for minor aggregation after reconstitution is indicated in the DLS-derived size distribution. On the other hand, there is no significant change in the SPR peak $\lambda_{\max }$ at $515 \mathrm{~nm}$ following reconstitution, though the peak magnitude decreases, indicating some loss of material. The results suggest that most of the dendron conjugated GNPs retain their pre-lyophilization singly-dispersed state. This is remarkable when compared to the cit-GNPs, which, when subjected to the same process, result in substantial agglomeration (clearly apparent by the color change in Figure 7). 

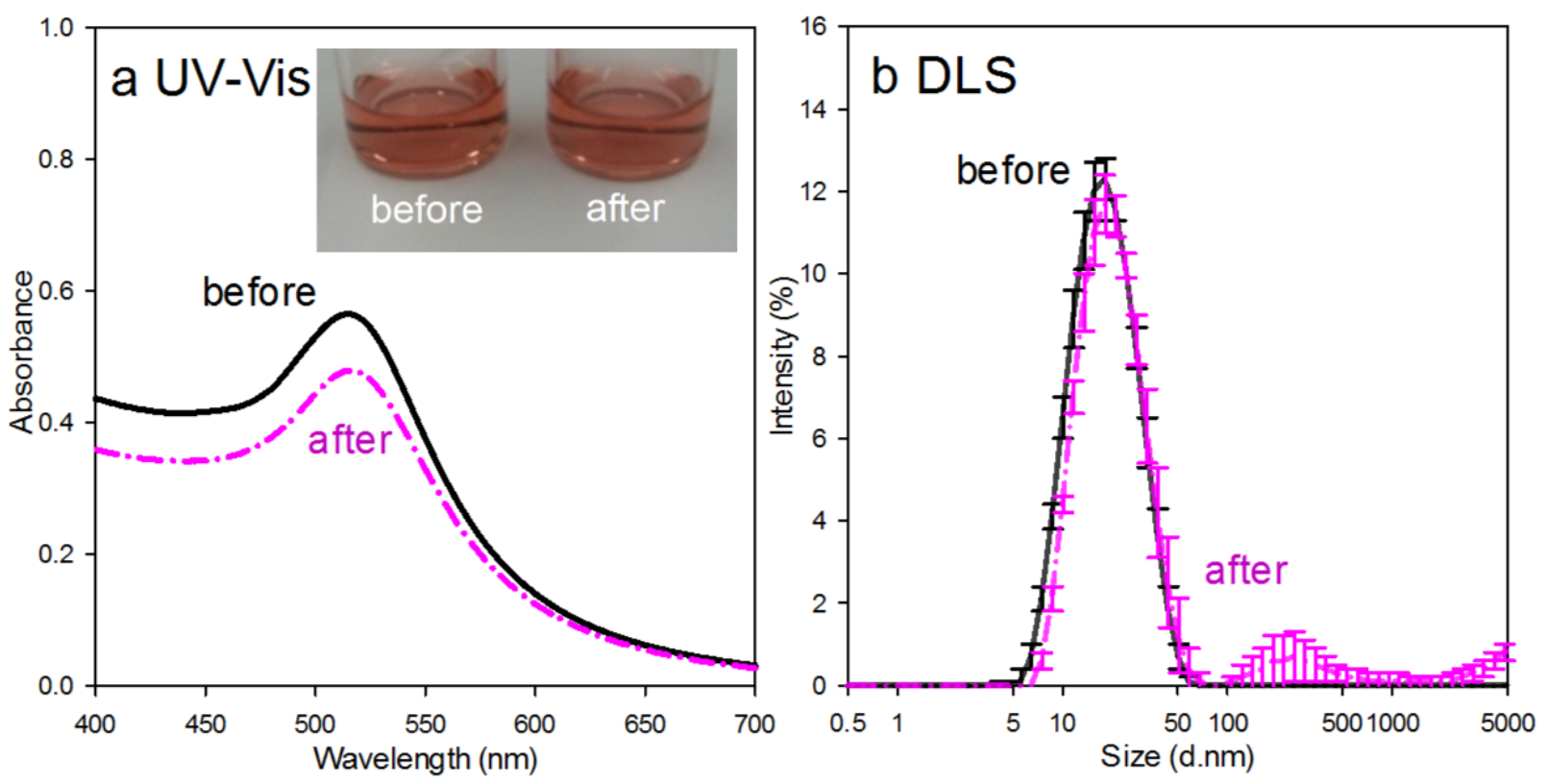

Figure 6. Comparison of (a) UV-Vis absorbance spectra and (b) DLS derived intensity-weighted size distributions for dendron-GNPs before (solid line) and after (dotted line) a lyophilizationreconstitution cycle. Inset shows characteristic translucent red color associated with stable unaggregated GNPs in this size range. ${ }^{8}$

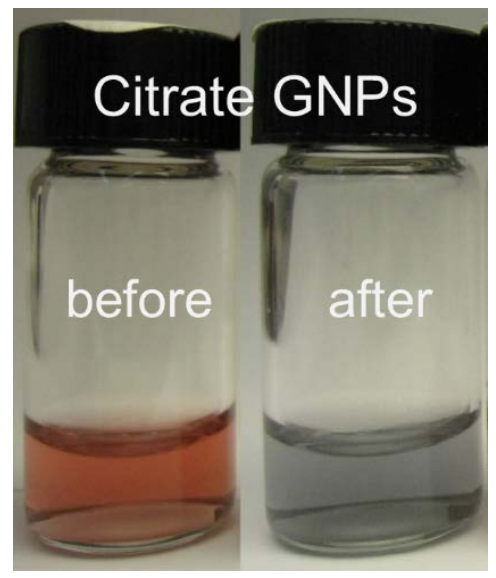

Figure 7. Before and after of lyophilization process for citrate GNPs. ${ }^{7,8}$

\section{Acronyms/Abbreviations}

aq

GNP

BSA

DI aqueous

gold nanoparticle

bovine serum albumin

deionized 


$\begin{array}{ll}\begin{array}{l}\text { DLS } \\ \text { DMEM }\end{array} & \begin{array}{l}\text { dynamic light scattering measurement or instrument } \\ \text { cit }\end{array} \\ \text { citrate stabilized } \\ \text { NCL } & \text { Nanotechnology Characterization Laboratory } \\ \text { NIST } & \begin{array}{l}\text { National Institute of Standards and Technology } \\ \text { phosphate buffered saline }\end{array} \\ \text { PBS } & \text { polydispersity index } \\ \text { PdI } & \text { polyethylene glycol } \\ \text { PEG } & \text { polymethylmethacrylate } \\ \text { PMMA } & \text { standard operating procedure } \\ \text { SOP } & \text { surface plasmon resonance } \\ \text { SPR } & \text { Ultraviolet-visible measurement or instrument } \\ \text { UV-Vis } & \end{array}$

\section{References}

1. D. A. Giljohann, D. S. Seferos, W. L. Daniel, M. D. Massich, P. C. Patel and C. A. Mirkin, Angewandte Chemie-International Edition, 2010, 49, 3280-3294.

2. R. Shukla, V. Bansal, M. Chaudhary, A. Basu, R. R. Bhonde and M. Sastry, Langmuir, 2005, 21, 10644-10654.

3. S. Eustis and M. A. El-Sayed, Chemical Society Reviews, 2006, 35, 209-217.

4. G. K. Min, M. A. Bevan, D. C. Prieve and G. D. Patterson, Colloids and Surfaces A-Physicochemical and Engineering Aspects, 2002, 202, 9-21.

5. $\quad$ S. Link and M. A. El-Sayed, Journal of Physical Chemistry B, 1999, 103, 4212-4217.

6. V. A. Hackley and J. D. Clogston, in NIST - NCL Joint Assay Protocol PCC-1, National Cancer Instititute, Nanotechnology Characterization Laboratory http://ncl.cancer.gov/working assaycascade.asp; http://dx.doi.org/NIST.SP.1200-6 2008.

7. T. J. Cho, R. A. Zangmeister, R. I. MacCuspie, A. K. Patri and V. A. Hackley, Chemistry of Materials, 2011, 23, 2665-2676.

8. T. J. Cho, R. I. MacCuspie, J. Gigault, J. M. Gorham, J. T. Elliott and V. A. Hackley, Langmuir, 2014, 30, 3883-3893.

9. T. J. Cho, J. M. Pettibone, J. M. Gorham, T. M. Nguyen, R. I. MacCuspie, J. Gigault and V. A. Hackley, Langmuir, 2015, 31, 7673-7683.

10. R. H. Garrett and C. M. Grisham, Biochemistry, Saunders College Publishing, Fort Worth, 1999.

11. L. Elsner, Journal fur Praktische Chemie, 1846, 37, 441-446.

12. C. A. Challener, Biopharm International, 2017, 30, 32-35.

13. R. I. MacCuspie, A. J. Allen, M. N. Martin and V. A. Hackley, Journal of Nanoparticle Research, 2013, 15.

14. D. E. Koppel, Journal of Chemical Physics, 1972, 57, 4814-4820.

15. K. L. Kelly, E. Coronado, L. L. Zhao and G. C. Schatz, Journal of Physical Chemistry B, 2003, 107, 668-677. 